(4) 'The polymerization of the above-mentioned propiolic acids.'

(5) The anhydrides of meta- and para-phthalic acids.

BROWN UNIVERSTY, PROVIDENCE, R, 1 .

[CONTRIBUTIONS FROM THLE CHEMICAL, [.ABORATORY GF THE UNIVERSITY OF ILLINOIS.]

\title{
METHYL MESOXALATE AND SOME OF ITS REACTIONS.
}

BY RICHARD SYDNEY CURTISS ANI PAET, T. TARNOWSRl.

Received Julle 12, $190 \mathrm{~h}$.

Several methods have been described for making methyl mesoxalate, but all are expensive in time and material, and leave much to be desired as to yield. Bouveault and Wahl" prepared ethyl and methyl mesoxalate by the reaction of nitrogen peroxide and ethyl and methyl isonitrosomalonate at a low temperature. Their yields were low, being between 34-40 per cent., most of their product being probably saponified by the action of water and the nitrogen peroxide used. Willstatter and Hottenroth ${ }^{3}$ prepared the substance by heating methyl bromnitromalonate in a distilling flask. The distillate consisted of anhydrous methyl mesoxalate which, with water, gave methyl dihydroxymalonate.

In the different experiments tried with the oxides of nitrogen and malonic esters various products have been obtained.

Conrad and Bischoff ${ }^{4}$ made ethyl isonitrosomalonate by treating the sodium salt of ethyl malonate in alcoholic solution with the red oxides of nitrogen.

Bouveault and Wahl" made methyl isonitrosomalonate by passing a current of methyl nitrite through a methyl alcohol solution of the sodium salt of methyl malonate P. T. Muller ${ }^{6}$ made the sane product by saponifying a methyl alcohol solution of nitrosocyanacetic ester with dry hydrochloric acid gas.

Traube $^{7}$ treated malonic ester with nitric oxide in presence of sodium ethylate and formed what he called oxazomalonic acid,<smiles></smiles>

One of us $^{8}$ treated ethyl malonate with nitrous anhydride at a low

${ }^{1} \mathrm{Mr}$. Dolt has just noticed that a specimen of piperonylpropiolic acid on exposure to light lost the power of reducing alkaline permanganate. This may prove to be the same naphthalene acid he obtained on heating with acetic anhydride.

2 Bull. soc. chim. [3], 29, 963 .

${ }^{3}$ Ber., 37, 178r.

4 Ann. Chem. (Liebig), 209, $2 \mathrm{II}$.

'Bull. soc. chim., 29, 96.

- Ann. chim. phys. [7], r, 536 .

${ }^{7}$ Ber., 28, I795.

${ }^{8}$ Curtiss, Am. Chem. J., 35, 477. 
temperature and obtained a yield of 90-95 per cent. of ethyl dihydroxymalonate. In view of the difficulties of making methyl mesoxalate, it was of interest to see if the above methods would yield as satisfactory results with methyl malonate as with the higher homologue. The results have shown this to be the case. A yield of $80-85$ per cent. of methyl mesoxalate has been otained easily, and other facts of interest noted.

Curtius $^{1}$ found that hydrazine hydrate reacts with ketones in neutral solutions to form unstable compounds of the type of hydrazones,

$$
\text { a. } \mathrm{R}_{2}=\mathrm{C}=\mathrm{N}-\mathrm{NH}_{2} \text {, }
$$

which, with water, readily pass into azimethylen derivatives,

$$
\text { b. } \mathrm{R}_{2}=\mathrm{C}=\mathrm{N}-\mathrm{N}=\mathrm{C}=\mathrm{R}_{2} \text {. }
$$

These compounds are readily decomposed by mineral acids into hydrazine and the ketone.

Curtius and Lang ${ }^{2}$ obtained hydrazipropionic acid,<smiles>CC(C)(C(=O)O)C1NN1</smiles>
from $\alpha$-oxopropionic acid and hydrazine hydrate. This was oxidized by mercuric oxide to diazopropionic acid. We were interested to see if this reaction could be carried out on methyl mesoxalate to give hydrazo malonic ester and from this diazomalonic ester, $\underset{\mathrm{N}}{\mathrm{N}}>\mathrm{C}\langle\mathrm{COOR}$, a substance which has long been sought for in vain.

Bulow $^{3}$ found that saponification takes place when the ester of methyl malonic acid is treated with hydrazine hydrate and a dihydrazide is formed. We find, to our surprise, that a hydrazine solution reacting on methyl dihydroxymalonate gives neither of the above reactions but

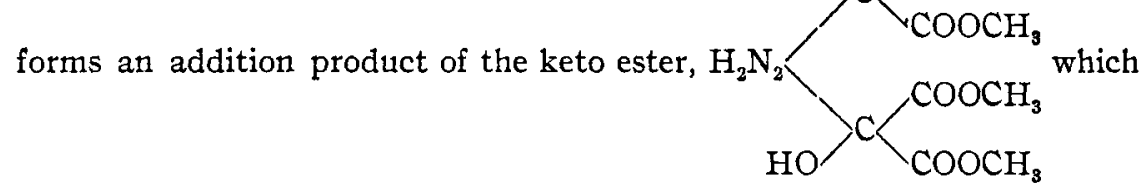

readily dissociates in water to the original constituents. Such addition products of substituted amines on ketone carbonyl groups are rarely met with in the literature. They may be considered as the unstable intermediate addition products in the reaction of all amines on aldehyde or ketone carbonyl groups and which, by loss of water, give the usual stable

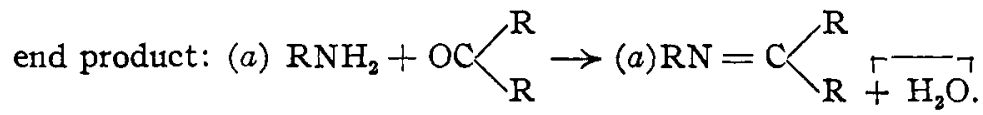

1 J. prakt. Chem., 44, 161-166.

Ibid., 44, 554 .

Ber., 39, 3372 . 


\section{Experimental.}

Preparation of Methyl Dihydroxymalonate.-Nitrous anhydride was generated by the action of nitric acid (sp. gr. I.42) on arsenious oxide. It was dried by passing it through a liter flask to condense moisture, and then through a tube containing phosphorus pentoxide. A rapid stream of this gas was passed into 25 grams of methyl malonate in a roo cc. distilling flask and cooled in a freezing mixture. It dissolved rapidly, and the liquid assumed a pale green hue, which became darker and almost opaque at the end of the operation. In one and a quarter hours 36 grams of gas had been absorbel. The flask was then packed in ice and kept at $0^{\circ}$ for 24 hours. It slowly loses gas, 3.5 grams in 4.5 hours, at which time the oil weighed 57.5 grams. Most of the remaining gas was removed by passing air through the solution at reduced pressure. During this process 12.5 grams of crystals of methyl dihydroxymalonate separated and were filtered off on the pump.

Ine green mother liquor from these crystals was subjected to fractional distillation at reduced pressure, in order to obtain the ketone ester, $\mathrm{O}=\mathrm{C} \angle \mathrm{CO}_{2} \mathrm{CH}_{2}$, and the other products of the reaction.

After the gas is freed entirely of oxides of nitrogen it is of a pale yellowgreen color.

The distillation was usually accompanied by two short periods of violent gaseous decomposition, the first occurring after the oil had been quietly distilling at about $70^{\circ}-75^{\circ}$ at $20-30 \mathrm{~mm}$. pressure, and the second toward the last of the process, -around $130^{\circ}-40^{\circ}$ at the same pressure. The gases evolved consisted (in the case of the ethyl ester) chiefly of nitrogen, nitric oxide and nitrogen peroxide and are formed by the decomposition of unstable nitrogen derivatives. At each period of decomposition the liquid reddens, but soon attains its former pale straw color, and the distillation proceeds quietly to the end.

In this distillation the first fraction, $40^{\circ}-70^{\circ}$ at $20-40 \mathrm{~mm}$. consisted chiefly of water, a little alcohol, acetic acid, and acetic ester, had a pungent odor, and continually gave off a gas. The remaining product, distilling from $105^{\circ}-125^{\circ}$ at $20-30 \mathrm{~mm}$., consisted of 19 grams of a thick green oil and was worked up for methyl dihydroxymalonate. When allowed to stand in the open air this oil absorbs water rapidly, becomes heated, and is changed into the crystalline methyl dihydroxymalonate,<smiles>CCOC(O)(C(=O)OC)C(=O)OC</smiles>

To facilitate this conversion, 75 per cent. of the theoretic amount of water necessary to change it into the dihydroxy ester was added. The temperature rose rapidly $40^{\circ}$, upon the addition of 
the water. After cooling in ice-water, 7.2 grams of crystals were filtered off. The total yield of the product in this particular experiment was 20 grams, or about 80 per cent. of the theoretical. By more careful manipulation it can be raised to $85^{-90}$ per cent.

The mother liquor (A) from these crystals weighed Io grams. This product contains other interesting compounds and will be described later in this paper.

The crude methyl dihydroxymalonate was recrystallized from dry ether and melted at $79^{\circ}$. It forms beautiful large plates, apparently monoclinic. It is very soluble in water, lowering the temperature decidedly. It dissolves easily in acetone, alcohol, and hot benzene. It is fairly soluble in ether and acetic ester, and dissolves slightly in benzene, chloroform, and carbon tetrachloride. It is insoluble in carbon bisulphide and in ligroin. Its solution reduces a warm ammoniacal solution of silver nitrate very quickly, the effect is slower when cold.

$$
\text { Preparation of Methyl Oxomalonate. - The ketone ester, } \mathrm{O}=\mathrm{C}\left\langle\mathrm{CO}_{\mathrm{CO}_{2} \mathrm{CH}_{3}}^{\mathrm{CO}_{3}}\right. \text {, }
$$

can be prepared by distilling the pure methyl dihydroxymalonate at reduced pressure. When heated above its melting point the elements of water dissociate and pass over. The keto ester then distils as a grassgreen oil. The first runnings recombine with moisture in the condenser and form crystals of methyl dihydroxymalonate. The second fraction boils at a constant temperature of $100^{\circ}$ (uncor.) under a pressure of $20 \mathrm{~mm}$. of mercury. with practically no decomposition, and is as pure an oxomalonic ester as can be produced thus. ${ }^{1}$

Reaction of Hydrazine Carbonate on Methyl Dihydroxymalonate.-We have studied the action of solutions of hydrazine hydrate and hydrazine carbonate on methyl dihydroxymalonate, and have obtained in each case a white crystalline product.

2.9 grams of methyl dihydroxymalonate were placed in $1.2 \mathrm{cc}$. of 60 per cent. hydrazine hydrate and kept cool in ice-water. The temperature rose slightly, and, in about five minutes, white crystals were formed, which were filtered and washed with water, and ether. The yield was not over 60 per cent. and the product was slightly colored.

A strong tendency of the reaction products to polymerize into a red gum was noted. This impurity is very difficult to remove. We therefore substituted a solution of hydrazine carbonate with good results, the same product being formed, but with a much higher yield and degree of purity. Thus, 3 grams of powdered hydrazine sulphate were added to a solution of 6.6 grams of sodium carbonate in $4 \mathrm{cc}$. of water. This

'Since this work has been completed one of us has found that only with the strongest dehydrating agents can a perfectly pure, water-free product be obtained. 
clear solution was added to a solution of 6.74 grams of methyl dihydroxymalonate in $15 \mathrm{cc}$. of water. Carbon dioxide was evolved when the two solutions were mixed and a fine, white, crystalline precipitate formed in a few moments. The solution was chilled in ice-water for five minutes and filtered, washed well with cold water, then with methyl alcohol, and finally with cold ether. The product was pure white and finely granular. The yield was 6.3 grams from 6.74 grams of methyl dihydroxymalonate. This product is already quite pure. When recrystallized from methyl alcohol it melts at $154^{\circ}-155^{\circ}$ to a clear liquid, which turns yellow and gives off a gas. If heated higher, the liquid forms a gum, When cooled, a yellow crystalline substance forms in the upper part of the melting point tube.

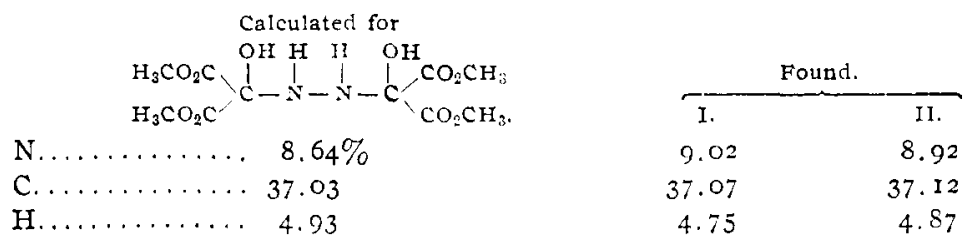

The formula given above is that of methyl hydrazotartronate. This substance is soluble in water, dilute sulphuric acid, 25 per cent. acetic acid, dilute hydrochloric acid, and sodium carbonate, when these solvents are heated and also in cold alkali hydroxide solutions. It is fairly soluble in hot solvents-acetone, acetic ester, 95 per cent. alcohol, and methyl alcohol. It is slightly soluble in benzene, carbon bisulphide, acetic ester, methyl alcohol, 95 per cent. alcohol and in water when used cold. It is insoluble in ether and acetone. It reduces an ammoniacal silver nitrate solution strongly when heated; slowly, if cold. Mercuric oxide is slowly reduced after acting a long time.

Phosphorus pentachloride reacts with the thoroughly dried substance, liberating voluminous quantities of hydrochloric acid, thus proving the presence of hydroxyl groups. Phosphorus pentoxide mixed with the white crystals slowly convert them into a yellow, crystalline compound, probably an azimethylen derivative. This has not yet been studied.

The above structure and name has been tentatively given to this hydrazine derivative of methyl dihydroxymalonate, the other possible isomeric form being that of an asymmetrical hydrazine compound,

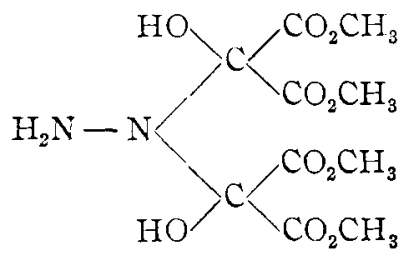


Action of Benzaldehyde on the Hydrazine Derivative.-When 7.08 grams of this substance are mixed with a dimolecular amount of benzaldehyde in $45 \mathrm{cc}$. of methyl alcohol, a reaction at once takes place, and a good yield is obtained of a substance crystallizing in long, beautiful, yellow needles. This has a melting point of $93^{\circ}$ when recrystallized from methyl alcohol. The analysis follows:

Calculated for, $\mathrm{C}_{8} \mathrm{H}_{6} \mathrm{CH}=\mathrm{N}-\mathrm{N}=\mathrm{CHC}_{6} \mathrm{H}_{8}: \mathrm{C}, 80.77 ; \mathrm{H}, 5.77 ; \mathrm{N}, 13.46$. Found: C, $80.58 ; \mathrm{H}, 5.83 ; \mathrm{N}, 13.64$.

The analysis, melting point and general properties show this substance to be benzalazine, described by Curtius. ${ }^{1}$ It is very soluble in ether, easily soluble in benzene, acetone, chloroform, carbon tetrachloride, hot methyl and ethyl alcohols, and in hot ligroin. It is insoluble in water and nearly so in cold ligroin. It distils in a test-tube at about $300^{\circ}$.

The mother liquor of the benzalazine crystals, when treated with phenylhydrazine, gave a fine, yellow precipitate, which gave the correct melting point, $163^{\circ}$, for the phenylhydrazone of mesoxalic acid. The water extract of the mother liquor of the benzalazine, after standing in a desiccator for two weeks, contained crystals. These melted at $79^{\circ}$ and, with hydrazine carbonate solution, regenerated the original hydrazine derivative of $\mathrm{m} . \mathrm{p} .155^{\circ}$, thus proving its identity as methyl dihydroxymalonate.

Owing to the ease with which this substance dissociates into hydrazine and mesoxalic ester it is impossible to determine whether or not the initial action of benzaldehyde on the hydrazine group precedes its dissociation from the mesoxalic radicle.

The Action of Hydroxylamine on Methyl Dihydroxymalonate.-Victor Meyer $^{2}$ made isonitrosomalonic acid by treating mesoxalic acid with hydroxylamine.

Methyl isonitrosomalonate, as made by Beauveault and Wah1, ${ }^{3}$ by the action of methyl nitrite on methyl malonate in methyl alcohol solution, crystallizes in long, white needles, melting at $66-67^{\circ}$. In order to obtain this oxime for study, an attempt was made to make it in the following manner: Ten grams of methyl dihydroxymalonate were treated with a solution of 4.76 grams of hydroxylamine hydrochloride and 3.66 grams of sodium carbonate dissolved in $9 \mathrm{cc}$. of water. When the two solutions are mixed, some heat is produced. It was warmed for five minutes to $100^{\circ}$ and then cooled, when a colorless oil separated out. It was extracted with ether, washed with water and dried over calcium chloride. Seven grams of a glycerol-like sirup, miscible with

$1 \mathrm{~J}$. prakt. Chem., 39, 44.

Ber., I6, 162т.

s Rull ene thim. 20. 062 . 
water, was obtained. When distilled in a vacuum a gaseous decomposition results. This ceases and is followed by a distillate at $165^{\circ}-169^{\circ}$ and $45 \mathrm{~mm}$. pressure. This clear, thick, oily product slowly crystallizes in long, colorless needles after standing a long time at $10^{\circ}$. These crystals melt at $64^{\circ}$. In addition to their solubility in water, and ether, and the formation of a yellow solution with alkalies, as mentioned by Beauveault and Wahl, ${ }^{1}$ we note that the substance is easily soluble in acetone, acetic ester, chloroform, alcohol and methyl alcohol; it is fairly soluble in benzene, slightly soluble in carbon tetrachloride, and insoluble in ligroin. It does not reduce a hot ammoniacal solution of silver nitrate. An odor of hydrocyanic acid is noted when it is boiled with water, as mentioned by Victor Meyer ${ }^{2}$ and Baeyer ${ }^{3}$ in the case of isonitrosomalonic acid under similar conditions. This substance agrees in melting point and other properties with those ascribed to the oxime of methyl oxomalonate and is without doubt identical with it.

A study of the Products in the Mother Liquor from the Mesoxalic Ester Crystals.-When methyl dihydroxy malonate is made in the manner described herein there remains in the green mother liquor (A, p. 1267) from the crystalline product taken from the distillate a complex mixture of unstable, oily esters having an acid character and containing nitrogen. This acid oil has been quite extensively studided, but owing to its instability and the explosive character of its salts, satisfactory analytical figures have not as yet been obtained. A brief outline only of this work will be given at this time.

When the mother liquor oils are washed with water to remove soluble acids, there remains a light green, insoluble oil of acid properties-a mixture of esters. This gives, with alcoholic potash solutions at low temperatures, a beautiful yellow potassium salt. This salt decomposes around $230^{\circ}$ after it has been recrystallized from go per cent. alcohol. The analyses show $19.4^{2}$ per cent. of potassium, and as the figures for a potassium salt of the oxime of methyl oxomalonate, $\mathrm{KON}=\mathrm{C}\left\langle\begin{array}{l}\mathrm{CO}_{2} \mathrm{CH}_{3} \\ \mathrm{CO}_{2} \mathrm{CH}_{3}\end{array}\right.$ are 19.59 per cent., it was at first supposed that this was the structure of the substance. The salt explodes when heated in a test-tube or on platinum--turns a darker color in sunlight-and yields a nearly colorless, thin oil when treated in ethereal suspension with dilute acids. The free acid thus purified is unstable and readily loses oxides of nitrogen in a desiccator. From it a potassium salt can be made which decomposes at $2.30^{\circ}$ and is identical with the original one. From it has also been made

'Bull. soc, chim., 29, 90́2.

2 Ber., I6, I62 1.

s Ann. Chem., I31, 293. 
an ammonium salt. This sublimes around $100^{\circ}-25^{\circ}$ and decomposes, when quickly heated, at $187^{\circ}-8^{\circ}$. The latter salt can be changed by silver nitrate to a yellow silver salt. It also yields a yellow salt with a dilute hydrazine hydrate solution. All these salts form beautiful yellow crystals and are explosive when heated.

This acid oil, while itself nearly colorless and insoluble in water, colors the water an intense yellow green and stains the skin a permanent yellow, like picric acid. It decomposes suddenly when heated to $120^{\circ}-$ I $30^{\circ}$, liberating red oxides of nitrogen.

The study of these acid oils, as well as of those formed in making ethyl mesoxolate, is under way and yielding interesting results.

One of us has also succeeded in preparing for the first time a perfectly pure anhydrous methyl oxomalonate. This substance has an unexpected intensity of reaction on the $\alpha$-carbonyl group and a number of interesting addition products have already been made and studied.

URBANA, ILl., June 2, I908.

\section{NOTES ON THE ROESE METHOD FOR THE DETERMINATION OF FUSEL OIL, AND A COMPARISON OF RESULTS BY THE ALLEN-MARQUARDT METHOD.}

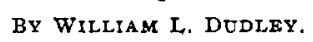

There has been considerable discussion in the Journals concerning the comparative merits of the Roese and the Allen-Marquardt methods for determining fusel oil in whiskies. For the past twelve months certain investigations have been made here which compelled a critical study of these methods, and our observations may be of service to others.

While these methods determine the higher alcohols, the results also include to some extent other constituents of the whiskey which with the higher alcohols have borne the name of "fused oil." This is especially true of the Roese method which doubtless determines many things beside the higher alcohols, and the results run much higher than those obtained by the Allen-Marquardt method, which in turn are probably somewhat low.

Discrepancies in results and many controversies which have arisen, are due in a measure to the variations in the technique practised by the analysts. Attention to minor details in these methods is absolutely necessary and the ordinary analytical procedure will not suffice. This is especially true of the Roese method. There is no description of this method given anywhere that we have seen which will give concordant results, if practised as an analyst would ordinarily follow a method. In fact, the Roese method if followed as described, is absolutely worthless. In this paper it will be our endeavor to point out the sources of 